\title{
Smart Care Spaces: Pervasive Sensing Technologies for At-Home Care
}

\section{Thomas", A.M., Moore, P., Evans, C., Shah, H., Sharma, M.}

Faculty of Technology, Engineering and the Environment, Birmingham City University, 1 Curzon Street, Birmingham, B4 7XG, UK.

E-mail: portablecomputing@googlemail.com

E-mail:ptmbcu@gmail.com

E-mail: cain.evans@bcu.ac.uk

E-mail: hanifa.shah@bcu.ac.uk

E-mail: mak.sharma@bcu.ac.uk

*Corresponding author

\section{Mount, S.}

School of Technology, University of Wolverhampton, Wulfruna Street, Wolverhampton, WV1 1LY, UK.

E-mail: s.mount@wlv.ac.uk

\section{Xhafa, F.}

Department of Software, Technical University of Catalonia, C. Jordi Girona, 1-3, 08034, Barcelona, Spain.

E-mail: fatos@lsi.upc.edu

\section{Pham, H.V.}

Soft Intelligence Laboratory, Graduate School of Science and Engineering, Ritsumeikan University, 1-1-1 Noji-higashi, Kusatsu, Shiga, 525-8577, Japan.

E-mail: haivnu@yahoo.com

\section{Barolli, L.}

Department of Information and Communication Engineering, Faculty of Information Engineering, Fukuoka Institute of Technology, 3-30-1 Wajiro-higashi, Higashi-ku, Fukuoka 8110295, Japan.

E-mail: barolli@fit.ac.jp

\section{Patel, A., Wilcox, A.J., Chapman, C., Chima, P.}

Faculty of Technology, Engineering and the Environment, Birmingham City University, 1 Curzon Street, Birmingham, B4 7XG, UK.

E-mail: asma.patel@bcu.ac.uk

E-mail: tony.wilcox@bcu.ac.uk

E-mail: craig.chapman@bcu.ac.uk

E-mail: parmjit.chima@bcu.ac.uk

Abstract: Society is experiencing an ageing demographic, coupled with increasing prevalence of Alzheimer's and Dementia conditions, expected to cause explosive increases in healthcare costs. Therefore, there is currently a need to develop pervasive technologies that allow monitoring of patients at home, where medically permissible, in order to reduce pressures on formal healthcare spaces. Those 'smart care spaces' require use of sensors and intelligent computer systems in order to support the needs of the cared-for, carers and medical personnel. In so doing they can ensure quality-of-life through comfort and adequate medical-monitoring, as well as providing significant data for on-going medical evaluation and diagnosis. This requires two main elements of sensing: sensors to monitor the care environment and patient-mounted sensors to monitor physiological parameters. Therefore, this paper considers technological options available for such monitoring and provides examples of their use. It will be concluded that cost-effective solutions are available for development of smart care space monitoring, to help ensure that associated computer systems maintain an appropriate level of intelligence. 
Keywords: Smart care spaces, e-health, sensors, aging, dementia, care, ad-hoc, ubiquitous.

Biographical notes: Andrew Thomas is a Research Fellow with an academic interest in sensors, sensing systems, sensor data interpretation and data visualization, including development of sensor circuits and associated computer systems. He holds a $\mathrm{PhD}$ in development of novel electromagnetic sensing techniques for soil analysis and monitoring. Philip Moore is a researcher currently completing a $\mathrm{PhD}$, his research interests include personalization, intelligent contextaware systems and ontology-based context modeling. Cain Evans is a Senior Lecturer in computer science with a special interest in enterprise architecture and systems, intelligent systems, intelligent marketing ontologies and eHealth. Hanifa Shah is an Associate Dean for Research with interests in utilizing technology for health care. Mak Sharma is head of the School of Computing, Technology and Networking and has a deep interest in networking and CISCO accreditation teaching.

Sarah Mount is a senior lecturer in computer science with interests in the Internet-of-Things and affective computing. Fatos Xhafa is a Professor in computer science with interests including parallel and distributed algorithms, combinatorial optimization, network systems and distributed programming. Hai Pham is a lecturer with specific interests in information science. Leonard Barolli is a Professor with research interests that include sensor networks, mobile communication systems, intelligent algorithms, agent-based systems and grid and Internet computing. Asma Patel is a PhD student with interests in use of mobile devices and RFID for health informatics. Anthony Wilcox is a senior lecturer specializing in electronics and robotics. Craig Chapman is a Professor of Knowledge-Based Engineering and Parmjit Chima is head of the School of Engineering, Design and Manufacturing Systems.

\subsection{Introduction}

\subsection{Background}

Currently society is witnessing a changing demographic toward higher percentages of persons over 65, a statistic that is accompanied by increased needs for healthcare. Pressures on formal care spaces are limited, and many people prefer the dignity and familiarity of being treated in their own homes (or other low-supervision settings). Also, many people live in remote settings with limited access to formal care institutions, and so may have little other option than to be cared for in that manner. This brings a need for greater monitoring of home 'smart care spaces' in a cost-effective manner that helps ensure comfort and medical needs are catered for, while providing carers and clinicians with data on which to base interventions and diagnoses.

Therefore, this paper considers the sensor technologies available for inclusion in such spaces, with a bias toward monitoring aspects that can be considered to be a priority for people experiencing age-related physical and cognitive decline (PCD). For them, the primary goals of at-homemonitoring are retention of familiar living arrangements, reduced premature institutionalization, maximizing independence and minimizing one of their most significant health problems: increased accident potential.

This article is structured as follows: the system is introduced and monitoring for smart care is discussed, with consideration of accidents and falling (significant issues in eHealth monitoring scenarios). Sensing and the environment is discussed in Section 2, with consideration of sleeping parameters, monitoring through interaction, behavior and power usage, together with other related environment sensing. Person-mounted sensing is addressed in Section 3 with consideration of walking (gait), activities and falling, and physiological monitoring using body sensors. The article closes with conclusions in Section 4, the overall conclusion being that while it is apparent that much of the technology required to create smart care spaces already exists, further research is required to integrate them into a functional whole.

\subsection{A holistic framework for Smart Care Space technologies}

Smart Care Spaces require holistic designs providing synergy between three main components, as illustrated in Figure 1. Firstly, they require a system of sensors encompassing both environment and patient. Secondly, they require a gateway for transmission of captured data to a central point. Finally, they require a cloud-based system for processing those data for 'real-time' monitoring and datamining (e.g. 'big-data' solutions), including databases within and outside the cloud.

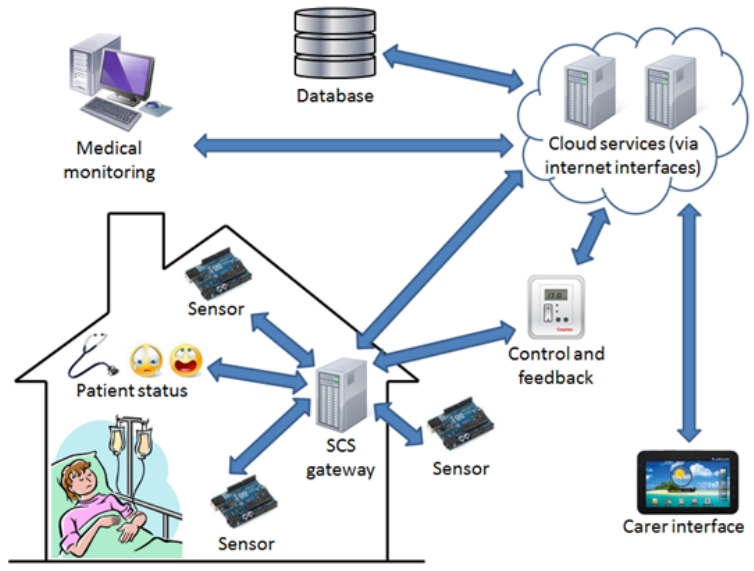

Figure 1. An overview of a Smart Care Space system. 
Optionally they may also incorporate control of care space systems from the cloud, including by clinicians and carers, as well as feedback to intelligent sensors. Due to comfort and privacy issues, there is also an emphasis on noninvasive sensors (e.g. sensing through the environment) and 'blind' camera technologies. Figure 1 also emphasizes a need for both local 'real-time' data processing and within the cloud. Local 'real-time' processing, with local decisionsupport and graduated interventions, is appropriate to, for example, agitation in dementia patients. Big-data, however, holds the prospect of identification of emergent properties and longer term prognoses, based on longitudinal monitoring. Even low-powered mobile devices are capable of local processing where a patients 'state' (more accurately the changes in a patients state: i.e. context) are measured based on the sensing data at times $t_{0}$ and $t_{1}$. Big-data solutions, however, require huge data storage and processing (we assume there will be large numbers of monitored patients) requiring extensive computing power separate to local care-space systems.

In this context it is tempting to consider patient status sensing as the central factor, but environment sensing is critical not just to ensure comfort but due also to the wideranging links between medical conditions and environment variables, including local weather conditions (see e.g. Thomas et al., 2013). Therefore, for a number of reasons, Smart Care Spaces represent not just sensing and information technology solutions, but instead require consideration as a holistic system if they are to succeed. So, in providing quantitative data, together with medical monitoring and carer interfaces, they aid integration of the two care needs identified by Roberts and Mort (2009): physical and social-emotional care.

Such a holistic approach is analogous to distributed intelligence, with obvious potential for use of emotions in machine-human interfaces (see e.g. Peter and Herbon, 2006). Therefore, as well as including human and machine intelligence, holistic smart care spaces may also be based on models incorporating emotional intelligence. For example, considering just anxiety as an emotional basis, together with scalable monitoring frequency of sensor data (i.e. activity) could allow intelligence based on valence and semiotics (see e.g. Perlovsky, 2006 for further discussion). This is illustrated in Figure 2, which shows an emotion-based valence for smart care systems that respects scalability (e.g. variations in sensor sampling frequencies), emotion and ICT-for-sustainability.

Under normal circumstances, the system remains vigilant, which essentially means that it requires context-relevant data-reception intervals that ensure potential problems are identified within the requisite time-window for the medical conditions being monitored (but without power wastage caused by over-sampling). When patients are absent, or at low risk of medical problems, the system could be calmer and less active, allowing power saving and so reducing sustainability implications. Then, when the patients' medical context demands increased sensor sampling rates, the system can be considered empathic in relation to the patient. In terms of self-preservation, unexpected reductions in activity (by sensor nodes and even in cloud-interface communications) would cause self-concern within the system, as it is likely to highlight technical faults.

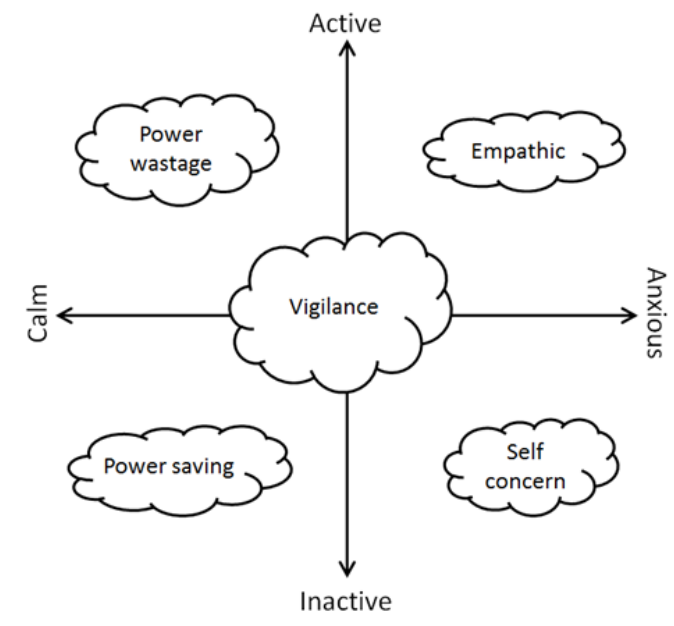

Figure 2. Smart care space status expressed as activity and anxiety valences.

Therefore, it is apparent that considering the smart care space in this way results in central and distributed elements, including autonomic aspects such as sensor nodes (which may rely on simplex or half-duplex communications for power saving) and networked elements (i.e. the gateway and cloud elements could be considered to have their own separable intelligences). For complete operability of smart care spaces within this paradigm, data from patient context and environment sensors are obviously critical as an 'intelligent' system is only as intelligent as can be achieved through the quality of data it processes. Therefore, this paper discusses the sensing aspects, the cloud aspect being largely outside of its scope.

As models of convergent technology, intelligent and holistic smart care systems are likely to rely more on mobile devices, such as smartphones and tablets. That is because, as well as providing significant processing power, sensors, low-power consumption and wireless communications, they are often being physically held by their users. That is very important for many reasons, including the potential for using them to obtain symptom-related data for longitudinal analysis, such as the prototype hand-trembling measurement application shown in Figure 3.

As well as measuring vibrations in the time-domain, the app provides magnitude data for discrete bins in the frequencydomain, which are linked both to age and medical condition progression. Smartphones have also been widely used in many areas of healthcare including tracking health information, making use of social-influence, improving data accessibility and to provide entertainment: for a full discussion see Klasnja and Pratt (2012). Therefore, it is essential that mobile technologies are incorporated into 
smart care space research, particularly as 3G/4G communications allow the opportunity to extend the carespace to outdoor excursions for patients.

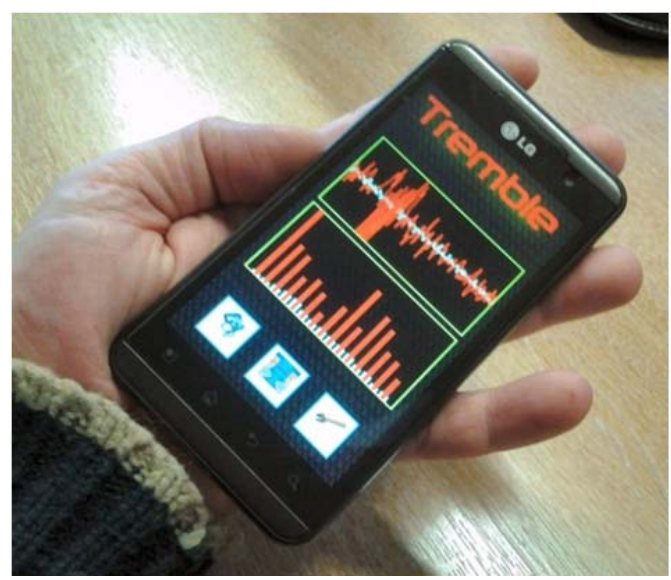

Figure 3. Measuring hand trembling using an Android smartphone.

So, overall, true smart care spaces require a holistic system comprising respect for environment data, patient-status data (including emotion data where possible) and intuitive interfaces, together with cloud systems capable of adequately processing those data and communicating relevant information to stakeholders. Much work has been undertaken on smart-home sensors, such as the 'smart condo' work of Stroulia et al. (2009) which integrated a wide range of sensors into graphical information systems and $3 \mathrm{D}$ visualization.

Of related interest is the work of Blackman et al. (2007) who investigated how people with mild to moderate dementia responded to outdoor environments: both a real and virtual town center. They found that outdoor activity had a beneficial effect and that, with adequate design and graphics-resolution, similar benefits may arise in virtual reality. As well as highlighting that text-based information is more appropriate for informing dementia sufferers (compared say to images) their work, together with that of Zeisel et al. (2003), indicates that use of interesting waypoints within sufferers homes makes them less likely to wander out of the care space. That correlates to use of sensors for location-context, and mobile devices for interaction between patients and their care spaces.

Of further relevance is the work of Biswas et al. (2006a and 2006b), which involved data collection and feature extraction using sensors in embryonic smart space applications. Their work is interesting in identifying the potential for independent living in smart spaces, although it was limited to laboratory environments. In terms of cloud architectures, the integration of smart homes into them has been covered by authors such as Yang et al. (2010), but in their case in terms of digitally interfaced consumer appliances. Similarly, it has been suggested that smart homes should have 'biological' intelligence, including through integration with the internet (see e.g. Wang, 2010).
However, as far as the authors are aware, no significant discussion has occurred in the literature relating to sensing aspects designed around holistic systems, encompassing the diverse aspects of smart care spaces.

\subsection{Monitoring for smart care}

Monitoring, as described herein, is a supportive tool for all participants in the care of our ageing population, allowing healthcare workers (medically-trained, or friends and relatives) to remotely interact with people under their care. Although potentially intrusive, it is a viable tool to support independent living and longitudinal monitoring, particularly as many people with PCDs prefer to live within a familiar environment rather than be institutionalized. This leads to a number of potential approaches toward maintaining their independence, using tools that allow maximisation of selfsufficiency. For instance, patient-mounted monitoring systems, which can partly be developed as mobile device apps, are unobtrusive and allow continued daily-living in the accustomed manner. A number of beneficial factors support this approach, notably reduced reliance on homecare-visits and a greater sense of independence, for patients, for as long as possible. Numerous vital signs can be monitored using mobile devices, such as skin conductivity, blood flow, patient orientation (e.g. lying down or standing), blood-sugar, gait, blood pressure and suchlike. These vital signs can easily be communicated using mobile broadband for carer peace of mind, and medical monitoring or even intervention.

Another form of monitoring discussed in this paper is potentially less intrusive monitoring of environmental variables and associated behavioural sensing within the home environment. A key feature of this home-monitoring is that people with PCDs, being cared for at home, can be monitored remotely as part of telemedicine. The technology infrastructure is already available and could be integrated with other home automation technologies and sensors. That could include shadow cameras (e.g. based on the Microsoft Kinect), voice-over-IP, and even using modern television technology to interact with friends and carers over video links. With express consent, to remove ethical difficulties, such technologies can support patients in maintaining connections to friends and family (and wider society), as well as supporting medical staff through a greater understanding of their patients needs. In so doing, this can bring the wider society around patients into their lives, compensating for their difficulty in going out from the home. It also allows those members of society to more easily have an active involvement in the day-to-day lives of people with PCDs.

As well as being used separately, patient-mounted and home-environment monitoring obviously provide enhanced function if combined and used with intelligent computer systems. Such integrated systems could be used to enhance carer peace of mind, as well as warning of potential dangers such as accidents, falls and even fires. They could then alert carers, medics or emergency services depending on the 
prevailing threat level. As well as home-monitoring, such systems could enhance the quality of life of care-home residents, where staffing levels may preclude full-time monitoring of their wellbeing. Thus, smart care space monitoring is not intended to replace human-to-human care where it exists, but to provide an enhancement to supplement that care, and enhance it also where full-time human care is precluded due to financial constraints and/or carer availability. It also provides an exciting opportunity to gain longitudinal data to aid in improving quality of life, based on a greater understanding of the impact of many PCD conditions.

\subsection{Accidents and falling}

According to the Alzheimer's Society (alzheimers.org.uk) there are a number of significant factors associated with accidents in people with PCD: declining sense of balance and reaction times; physical difficulties and mobility problems; diminished memory, judgement and awareness of danger; pressures-on and tiredness-of carers; and stress and confusion. Therefore, systems that monitor motion and activity, while supporting the activities of the cared-for, and assisting the workload of carers, must have significant potential. For instance, the potentially sedentary lifestyle requires monitoring of activity and effects on the circadian rhythm. Also, changes in cognitive ability can be monitored through interaction with care space system interfaces. In such cases changes can be detected through behaviouralteration, which could simply be achieved through energyuse measurements, or even through patient-mounted gait monitoring. Such patient-mounted systems can also provide activity data and 'person-down' alarms in case of emergencies, which can be considered important because one of the most important sensing needs for PCD patients is associated with the risk of falling.

Table 1. Dementia fall factors (after Shaw, 2003).

\begin{tabular}{ll}
\hline Fall factor & Risk factor \\
\hline Impaired gait and balance & $99 \%$ \\
Environmental hazards & $80 \%$ \\
Medication & $70 \%$ \\
Neurocardiovascular instability & $60 \%$ \\
Orthostatic hypotension & $40 \%$ \\
\hline
\end{tabular}

Falls are most likely in home settings, for people over 50 years of age, and particularly in males (Kool, et al., 2007). Dementia is a very significant factor also, with demented women having been reported as having more than double the risk of hip fracture compared to other $>85$ year old women (Johansson and Skoog, 1996). Consumption of even moderate amounts of alcohol significantly increases risk
(Kool et al., 2008) and, according to Khawandi et al. (2011), as many as a third of people over the age of 65 will suffer at least one fall per annum, while as many as 4 in 7 falls may be fatal in the over-70s (Atallah et al., 2012). For persons with dementia that incidence may increase to $70-80 \%$ (Shaw, 2003), due to gait and balance impairment (Shaw \& Kenny, 1998). The most significant risk factors associated with falls among dementia sufferers are illustrated in Table 1 , from which it can be taken that risks are associated with care space design, medication, other medical conditions and the effects of dementia itself.

\subsection{Sensing using the environment}

\subsection{Light, sleep and the Circadian Rhythm}

Light sensors have obvious advantages in monitoring, such as whether there is enough light to minimise accident potential both during the day and during night-time activity. However, they can also monitor night-time activity patterns, especially in combination with other devices such as proximity sensors. Those patterns can expose potential sleep issues, as people chronically departing from seven hours of sleep per day can be associated with increased mortality rates: both from the number of hours slept and, in the case of insomnia, from prescribed medication (Kripke et al, 2002). However, the optimum amount of sleep is agerelated (Klerman \& Dijk, 2008), may vary over short timescales, and may include daytime napping consistent with afternoon dipping of the circadian rhythm (Kloss et al., 2002). That may be an important factor in smart care space monitoring as the circadian rhythm varies between individuals, may on average be greater than 24 hours and can vary significantly between genders (Duffy et al., 2011).

An indication of the economic benefit of including sleep related factors in environmental monitoring is given by Engelhardt (1999): the direct economic cost of insomnia in the United States was estimated at \$13.9bn for 1995 alone. Sleep difficulties can be linked both to evening artificial light intensity and spectral composition, with increased effect associated with blue components (Santhi et al, 2011). Illustrated in Figure 4 are light level data for two rooms, one with natural, and the other artificial, lighting. As shown in Figure 4(a) brightness levels can vary significantly under natural light, the much brighter levels that could be associated with many medical problems being evident from the figure. Negative consequences of low light (e.g. Seasonal Affective Disorder) could also be monitored this way. The data of Figure 4(b) are based on a room with artificial lighting and patterns of activity can be determined from it.

Intelligent environments offer the potential for personalized interaction with the cared-for, including to monitor deterioration of medical conditions, humans often being the best sensors into their own states. For instance, in Dementia, simple 'clock-tests' are used (involving representing times on a clock face) (Scanlan et al., 2002), which could be used as a basis for automated testing. They also offer the 
potential for oral interaction between environment and cared-for leading to diagnosis and longitudinal monitoring, or even monitoring of emotional state based on affective spaces (see, e.g. Leon et al., 2007 and Bradley and Lang, 1994, for further details). For example, the Parkinson's Voice Initiative (www.parkinsonsvoice.org) claim significant accuracy of detection in Parkinson's Disease through analysis of patients' spoken words.
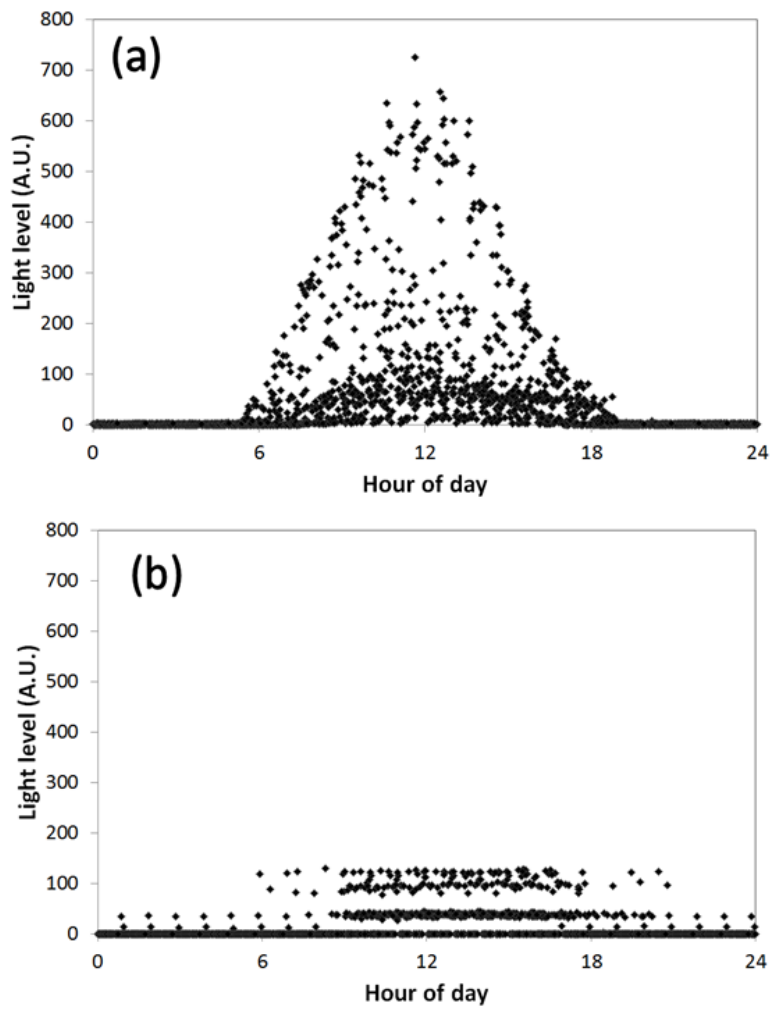

Figure 4. An example of light level monitoring over two rooms (a) with natural lighting and (b) with artificial lighting.

\subsection{Monitoring through interaction}

Such oral interaction may also provide a means to incorporate some social-emotional care as advised by Roberts and Mort (2009). For instance, Heerink et al. (2008) and Heerink et al. (2009) suggested a strategy for testing whether robots could be accepted by PCD people based on assessing user-perception of a robot. They tested this method using 'iCat', an information-giving robot, and suggest that robots with perceived extraverted social abilities are more likely to be accepted and reported as 'enjoyable' companions. However, adequate attention to detail in design is obviously important, as shown by the work of $\mathrm{Wu}$ and Miller (2005). Their study on the role of affective (i.e. emotionally engaging) technologies and care of PCD people, using a chatbot with a telephone interface, suggested that voice synthesis can be perceived as cold and insincere. Such interfaces to smart care spaces, for the cared-for especially, therefore need to be both intelligent and adequately engaging.
It should also be noted that interaction with smart care systems need not be simply oral or physical. For instance, EEG signals can be used to provide cognitive interfaces such as in the work of Hu et al. (2011) which also included use of EEG to identify chronic stress groups. Use of EEG as an interface has the added advantage of monitoring patientspecific conditions such as epilepsy (Schelter et al., 2006), depression and schizophrenia (Li et al., 2008). However, drugs are known to impact EEG data, to the extent that van Bemmel (1996) described '...remarkable changes in sleep polygraphic variables'. This makes interpretation more complex, although the data can be used to monitor condition-improvements during use of prescribed medication (Steiger and Kimuru, 2010). Also, where the ethical and intrusion-related concerns can be overcome, use of imaging techniques (including CCTV, Kinect, etc.) can provide significant signal data for monitoring emotional state (e.g. Vinciarelli et al., 2009).

Given the widespread adoption of mobile phones and tablets, an important area of interaction is through use of mobile applications and web pages. Figure 5 shows a prototype system able to access relevant data using RFID, barcodes and speech recognition. Current and historic sensor data are both easily accessible, together with simple visualisations. This tablet 'app' was developed with ease of use in mind, with only three buttons to change interaction mode (i.e. RFID, barcode, speech). The system has obvious advantages for carers in accessing environmental monitoring data, medication access logs, medical records and suchlike.

However, it can also provide the cared-for with a means to maintain some control over their environments, access data about care space features, provide feedback on their psychological/emotional status, and even allow them to access data about recent activity as an aid to memory. As will be discussed later, the same mobile devices' sensors can also be used to collect relevant data such as activity levels and location.

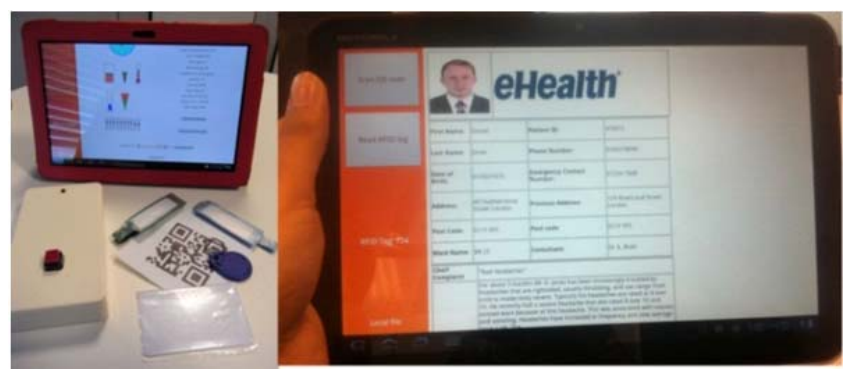

Figure 5. Mobile interface to sensors and care space data.

\subsection{Behaviour and power}

Significant behavioral information can be obtained through monitoring of electricity consumption (see e.g. Wood and Newborough, 2003). Even without smart-meter data, this can be simply and inexpensively accomplished through submetering (Berges et al., 2008). However, it has been 
cautioned that smart-metering systems could leak private and sensitive information, or unauthorized control could be gained, if not designed with care (Gao et al., 2012). It has been reported that electricity consumers have concerns about the detail and immediacy of smart-metering data, not just in terms of the potential for loss of privacy through realtime surveillance but also through the potential for its' use in planning burglary and assault (Krishnamurti et al., 2012). As an example, Figure 6 shows simple current-clamp and microcontroller power measurements during a weekend in a domestic residence. Use of an electric oven, evening television and intermittent refrigerator consumption are all evident, as well as strong short-duration peaks due to boiling water in a kettle.

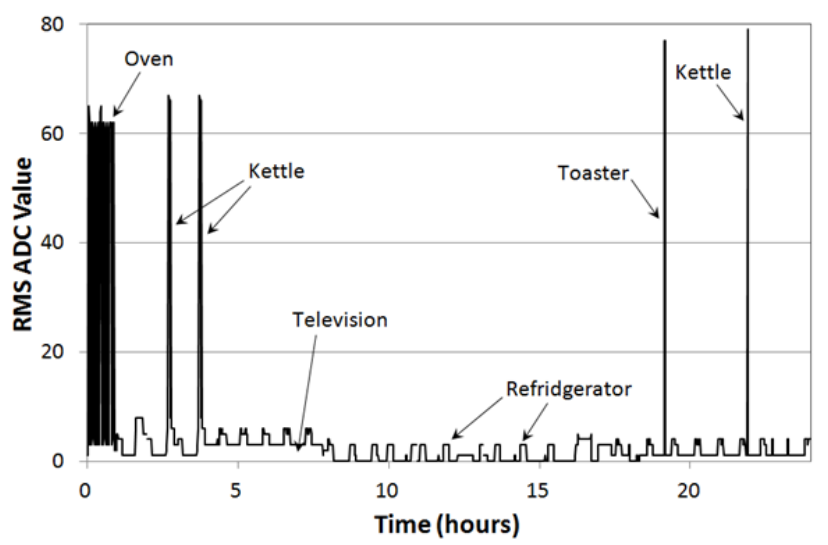

Figure 6. Microcontroller measurements of variations in domestic power consumption over a 48-hour period.

\subsection{Other environment sensing}

Disease-related malnutrition is an important problem in PCD people causing impaired muscle function, decreased bone mass, immune dysfunction, anaemia, decreased cognitive function, poor wound healing, and increased (c.50\%) hospitalisation (Baumeister et al., 2011). Such issues can easily be monitored through use of simple switches and proximity sensors within food preparation areas. Similarly, López-Nores et al. (2012) have explored the potential for use of internet-connected mobile devices to aid medication adherence. It has been stated that up to half of patients do not take medication in the manner prescribed (López-Nores et al., 2012). From a safety perspective, methane gas detectors are widely used and relatively inexpensive: they can also be used in conjunction with safety shutoff valves to minimize accident risks (Daniel et al., 2009).

Similarly, monitors such as smoke, carbon monoxide and flood detectors are pervasive and so are not discussed in depth herein, as are bed/chair occupancy sensors, flood detectors and suchlike widely used for conditions such as dementia. Simple bed and door sensors have been used by Rowe et al. (2009) that the authors claim to significantly reduce night time injuries and unattended home exits. It should also be noted that simple optical dust sensors are widely available that could be of significant benefit for people with conditions such as asthma and allergies. Also, simply monitoring weather may also help warn of asthma risk: rainy weather having been reported to create a washout effect for pollen, but with sudden storms potentially causing an increased risk of attacks (Ho et al., 2007; Pullimood et al., 2007).

\subsection{Person-mounted sensing}

\subsection{Gait sensing}

Sensors can be put to many uses when worn or carried including on-going monitoring of gait and balance deterioration, and detection of potential and actual falls. Accelerometer-based circuits have been researched for many years, such as in monitoring orientation, mobility, gait, energy expenditure and falls and stumbles (e.g. Mathie et al., 2001). Other systems, such as that of Khawandi et al. (2011) also allow fall monitoring, although the use of cameras could be considered invasive to privacy (which could potentially be overcome using 'shadow' data such as from a Microsoft Kinect). Interventions could follow from this, such as alterations to footwear and determination of the need for hip protection (Shaw \& Kenny, 1998). Important indicators of gait impairment include reduced walking speed, reduced step frequency and shorter step lengths (Shaw, 2003), and can be monitored at low cost through simple accelerometers and gyroscopes. As an example, Figure 7 shows frequency analyses of smartphone sensor data illustrating the ability to use such data to identify variations in walking pace. For patients with Dementia, variability in walking speeds has been shown to be related to progression of the condition (Kaye, 2008).
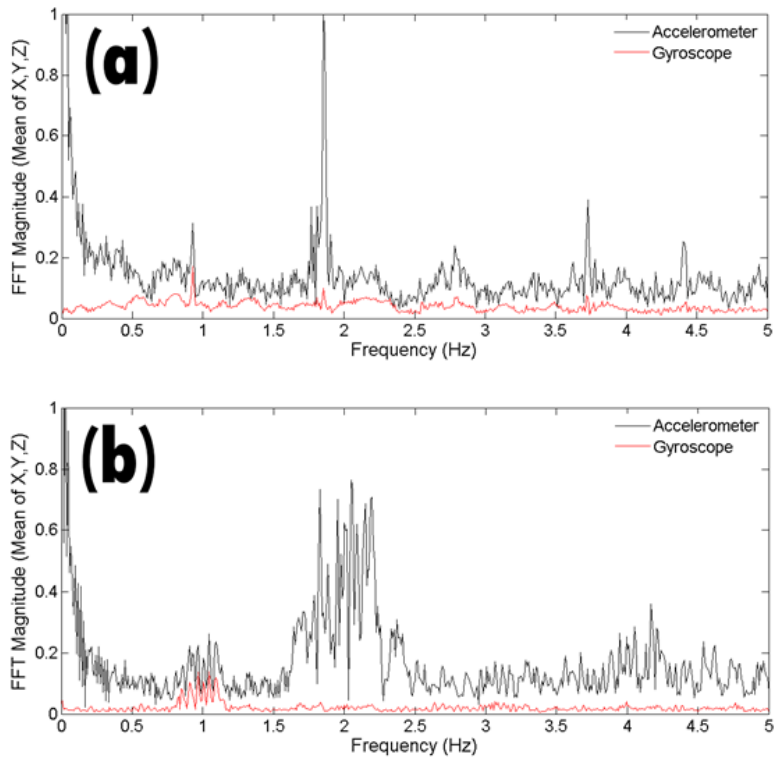

Figure 7. Frequency-domain analysis of walking (a) at a uniform pace and (b) at varying pace.

Use of mobile phones for such monitoring is advantageous in that patients are more likely to carry one because they 
form a convergent technology in a system with many additional uses. Where such convergence cannot be achieved, dedicated body-area sensors appear most likely to gain user acceptance if incorporated into clothing (e.g. Yanfen and Pu, 2011). Lee and Chung (2009), for instance, reported on a wearable wireless sensor-node (the 'smartshirt') incorporating accelerometers and an electrocardiogram (ECG) able to send data through a wireless network for viewing by medical personnel. This may be particularly important for dementia sufferers, as they may have limited tolerance toward devices attached to their person.

\subsection{Activity and fall monitoring}

Cameras, Microsoft Kinects, 3D scanners and suchlike (e.g. see Vinciarelli et al., 2009), offer potential for further activity monitoring, and even therapeutic activity, where body-sensors are inappropriate and camera-based techniques are considered acceptable. Smartphone, and simple body-area, sensors can also be used to detect falls and warn carers, but the context of a fall is different to walking. For instance, the frequency analyses of Figure 7 are based on a $10 \mathrm{~Hz}$ sampling rate, which is sufficient for activity monitoring, and reduces power consumption, but is unlikely to be adequate for fall detection. In a fall context much faster sampling rates may be appropriate but will impact on mobile equipment battery life. However, alternatives exist including use of activity data, such as the activity monitoring system of De et al. (2012) which incorporates behavioural analysis. For instance, the lowfrequency offset of accelerometer data in the time-domain, which represents device orientation, together with activity data, provides useful monitoring data. This is illustrated by Figure 8, using data from smartphone sensors, for normal research office activity over one hour. The occurrence, and magnitude, of activities are represented by variations in the accelerometer value over time, with orientation changes of the wearer being apparent at step-changes in mean values. As illustrated by Figure 9, the accelerometer data can be considered in terms of frequency components (here over ten second windows) that can provide further data on potential activities being undertaken.

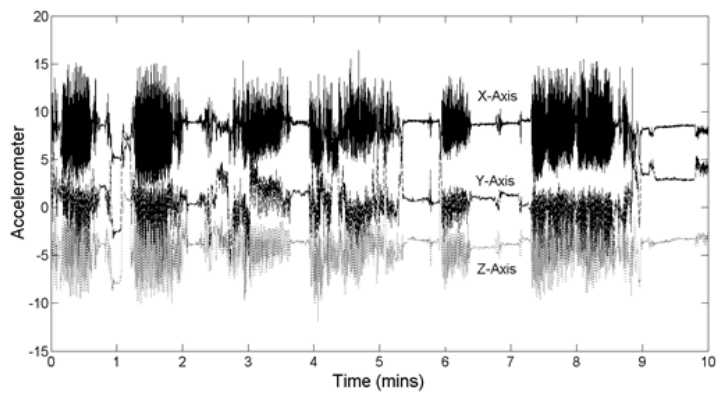

Figure 8. Use of an accelerometer to detect activity and orientation.

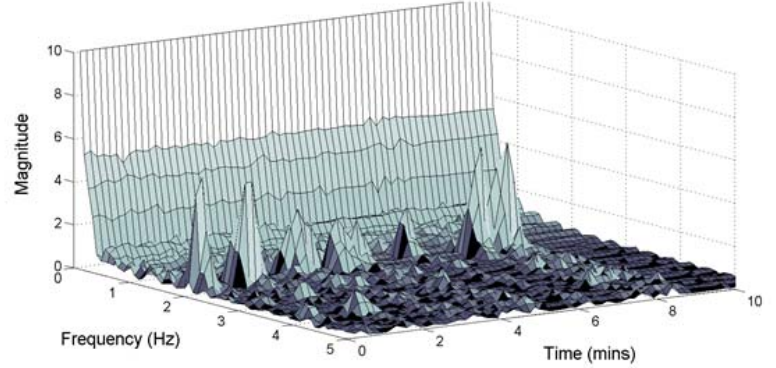

Figure 9. Frequency components of the data from Figure 8 at tensecond intervals.

From Figure 8 it is apparent that the likelihood of a fall can be estimated through detection of reductions in activity level and a change to non-vertical orientation. However, this method is crude as it does not account for other context factors: i.e. it is difficult to ensure those changes are not associated with normal activities such as sleeping in a bed or chair. This could be addressed partially through simple devices, such as pressure switches, built into chairs and beds. However, more sophisticated location context data is available through low-cost radio modules, such as XBee devices based on the ZigBee (IEEE 802.15.4) communications protocol. Under ideal conditions the signal strength data communicated by these modules can be used to accurately locate objects within a few centimetres (e.g. Pichler et al, 2009).

However, even under less ideal conditions they are still capable of providing useful location context (Chan, 2010), such as within which room a patient is located, and the approximate position within that room. They also have the advantage of being able to relay data from simple sensors. The location context can be used to estimate the probability that a patient is resting or sleeping, and therefore enhance other, cruder, indicators. In some patients, such as those with dementia, it could also provide indications of important symptoms (e.g. wandering or agitation: Shaw, 2003).

For all patients it can be used to develop activity patterns which can be used in intelligent cloud systems to detect abnormal behavior. For instance, unusually frequent detection that someone is visiting the bathroom may indicate medical problems. Also, forgetfulness is a common problem for people with PCD and dementia patients (Commissaris et al, 1996). Location data could provide them with a useful way to recall recent actions when necessary, or even provide a crude probability that a patient is accessing food and medication regularly, amongst many other possibilities. Also, in a home care environment physical activity may often be reduced. Activity and foodintake monitoring may therefore be useful in indicating insulin sensitivity and Type II diabetes (the risk potentially increasing as activity levels reduce: Brouwer et al., 2007). Similarly, Pasman et al. (2011) used galvanic skin responses (GSR) in deriving links between anxiety and balance in patients with and without Parkinson's Disease. 
Another technology that could be used for location purposes is RFID which is already in widespread use in hospitals and can be worn, incorporated into clothing or used within smart cards. It can, for instance, be used to store patient health records (Naszlady and Naszlady, 1998), or to monitor medication use and prevent duplicate prescription (Hsu et al., 2011). They can also be used to facilitate longitudinal healthcare information and track patient pathways through care (e.g. Marschollek and Demirbilek, 2006). It is also possible that RFID devices could be embedded in smart care spaces, such as in furniture, cupboards, medicine cabinets and suchlike, for activity and even patient-location. Furthermore, using re-writable tags it is possible to store recent activities for access by forgetful persons.

\subsection{Physiological body-area sensing}

Sensor systems mounted on the person are becoming pervasive in modern research, covering anything from simple sensors to complete biomedical systems for monitoring astronauts during extra-vehicular activity (Fei et al., 2010). Obviously, as all humans are contextuallydifferent, such systems should be contextually-aware in order for their data to be properly interpreted (Domingo, 2012). Many examples of patient mounted systems have been reported in the literature: for example Pandian et al. (2008) reported on their ECG inclusive 'smart-vest' for physiological monitoring. Rodrigues et al. (2011) also devised a body sensor network system, but interestingly providing biofeedback visualization, which may be of use in aiding patients to better cope with their conditions. Amongst many other examples is the chest-mountable 'smart-belt' of Sardini and Serpelloni (2010), which included measurement of ECG traces, heart-rate, respiratory-rate, body temperature, position and acceleration. Measurement of ECG data is now a relatively simple exercise due to the sophistication of modern microcontrollers, Figure 10 providing an example of such data.

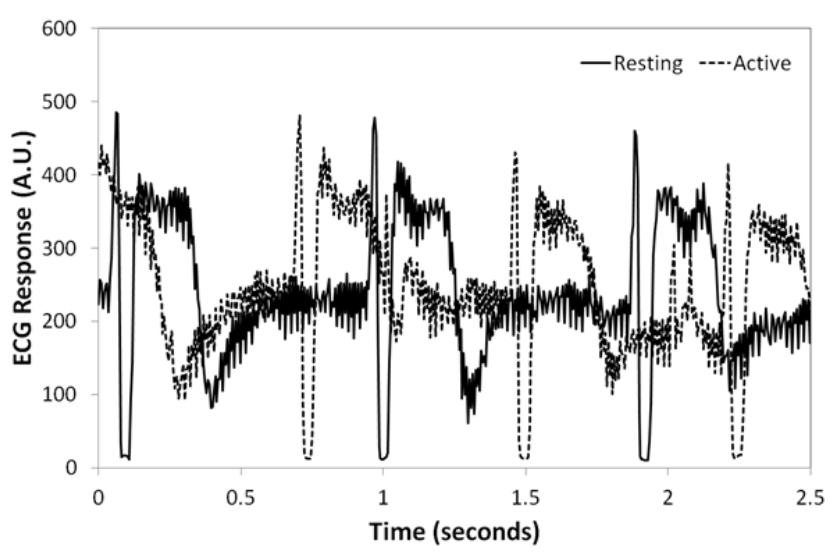

Figure 10. Example microcontroller ECG data capable of heartrate monitoring.

Sensors are also available for jointly measuring respiration and heartbeat, for example the work of Jiang et al. (2010) which allows separation of sensing into two data channels.
Chen (2011) developed a human pulse-monitoring system that is internet-connected for data analysis, storage and remote viewing. Body-mounted systems have also been developed for nutrition assessment, such as the work of Soller et al. (2002). Similarly, body mounted electrodes for tracking eye movements have been used for mental health monitoring (Vidal et al., 2011). A further area of potential is changes in the electrical properties of skin due to environmental conditions and external stimuli: such as through potential differences in sympathetic skin response and GSR measurements (see e.g. Arunodaya and Taly, 1995). Both are closely linked to sweat production (Ellaway et al., 2010) and so could be adopted for measurements of fevers, comfort and emotional response. In terms of dementia Eisenstein et al. (1994) reported that GSR could be used to detect small, early, changes in learning and memory caused by ageing and dementia, which appears to relate well to the findings of Zhang et al. (2012) in their consideration of correlates of skin conductance responses during cognitive tasks.

More recently, van der Broek and Westerink (2009) reported on the potential for use of GSR to add emotional insights to consumer products, an approach that could have many uses in smart care spaces. Even in coma patients, Daltrozzo et al. (2010) found that skin electrical measurements could detect some emotional response, although reducing with decrease in consciousness. In terms of the link to depression discussed earlier, and anxiety, sympathetic nervous system responses have been linked to EEG measurements (Papousek and Schulter, 2001). Also of interest is the work of Gulbandilar et al. (2008) who found relationships between skin electrical resistance and static balance in Type II diabetics, that of Røeggen et al. (2011) who researched links between it and infant anxiety and pain, and that of Nagai et al. (2004) who undertook research to allow GSR to be used for biofeedback as intervention for epilepsy.

Other examples include the work of Farella et al. (2010), who describe use of fixed and person-mounted sensors within a home monitoring system: their system included ZigBee-protocol communications and was intended to provide indoor tracking and warning of potentially harmful events. Of related importance is that Steele et al. (2009) found that people, such as those with PCD, are generally positive about the use of wireless sensors in their care, as long as the cost to them is low and they have a perceived high level of control over the sensors and systems (i.e. they do not see their personal decision making level eroded). Also, Fenza et al. (2012) considered means to personalize home and hospital body area networks and add contextawareness, including through use of semantics and fuzzy logic. Their system included patient-mounted sensors together with a context-matching system to link detected events to healthcare services.

As well as real-time monitoring, sensors in smart care spaces, including worn by patients, allow for gathering of longitudinal data for data-mining and baseline behavior 
modeling. For instance, Duchêne et al. (2007) describe methods of learning recurrent behaviour from multi-variate medical time-series data, to build behavioural profiles of peoples' day-to-day activities. Virone (2009) extended on general activity-determined behavioural analysis to specifically consider older generations. They not only considered determination of activities in day-to-day life, based on simulated data, but also consider how those patterns are disrupted when monitoring for Dementia.

The problem of wandering is widespread among some patient groups: one estimate being that $60 \%$ of people with Alzheimer's will wander from their homes per annum (Daniel et al., 2009). This has been addressed in the literature through electronic tagging which has been reported as effective and with limited ethical issues amongst relatives and carers (e.g. Miskelly, 2004). Given the low cost of radio-location (and GPS for longer distance wandering) devices it could therefore be considered a simple task to include tagging in body-mounted care-space systems. In fact, Lin et al. (2006) went further in their work to integrate GPS and RFID into geographical-information systems to prevent straying in dementia sufferers.

\subsection{Conclusions}

Ageing demographics, as well as the needs of people in remote communities, brings a market for cost-effective athome care monitoring-systems. This is particularly pressing in terms of supporting people with PCD, including those with dementia, who are at significantly higher risk of experiencing accidents and falls, and who may have cognitive decline requiring assistance with memory and everyday tasks. It is also important for their carers, who may face significant pressure, stress, and demands on their time that may be eased through up-to-date access to monitoring data. If those reasons were not enough, longitudinal monitoring of smart care spaces provides potential improvements to on-going diagnoses and may even illuminate new treatment options.

From a cost effective perspective, use of simple sensors can pay significant dividends. As an example, light sensors can provide data to aid in reducing accident levels, monitor night-time activities, and even aid in preventing sleep issues. That could be considered useful not only because insomnia brings large monetary costs, but also because quality of sleep is related to quality of life and lifeexpectancy. Also, behaviour-related data can provide clues to advancement of clinical conditions, as well as aiding those being cared for through provision of activity-tracking records for augmentation of memory. Those data could be obtained through many routes, including radio-location, RFID tagging, 'shadow' cameras and even monitoring of power consumption.

While data from the above can be obtained remotely from the cared-for, there is often a need to obtain data that can only come from patient mounted sensor systems. For instance, activity levels are best measured using body- mounted accelerometers and gyroscopes. In such circumstances viewing that monitoring as convergent with mobile devices has obvious advantages: many modern smartphones incorporate those sensors, can run internetlinked 'apps', and may be more accepted by users due to their potential to fulfil many other desired uses. Other sensors could be similarly, and inexpensively, used such as EEGs, ECGs, pulse-monitors, GSR and suchlike, all of which can even be simply interfaced to modern smartphones.

Even if only the above items were installed in smart care spaces then significant advances could be made in terms of meeting the needs of carers and cared-for. However, intelligent methods of accessing those data provide further enhancement, particularly when designed as part of holistic systems and potentially with the addition of emotional intelligence and intuitive interfaces. Mobile devices for system interfacing can be used to log activity data, but could also be used to allow input of psychological and emotion data. They can also allow interaction with smart care spaces, including accessing sensor and medical data, and even querying objects for forgetful residents. In conclusion, therefore, it is apparent that much of the technology required to create smart care spaces already exists, but further research is required to integrate them into a functional whole.

\section{References}

Arunodaya, G.R. and Taly, A.B. (1995) 'Sympathetic skin response: A decade later', Journal of the Neurological Sciences, Vol. 129, pp.81-89.

Atallah, L., Lo, B. and Yang, G.-Z. (2012) 'Can pervasive sensing address current challenges in global healthcare?', Journal of Epidemiology and Global Health, doi:10.1016/j.jegh.2011.11.005.

Baumeister, S.E., Fischer, B., Döring, A., Koenig, W., Zierer, A., Johns, J., Heier, M. and Meisinger, C. (2011) 'The geriatric nutritional risk index predicts increased healthcare costs and hospitalization in a cohort of community-dwelling older adults: Results from the MONICA/KORA Augsburg cohort study, 1994-2005', Nutrition, Vol. 27, pp.534-542.

Berges, M., Goldman, E., Matthews, H.S. and Soibelman, L. (2008) 'Training load monitoring algorithms on highly submetered home electricity consumption data', Tsinghua Science and Technology, Vol. 13(S1), pp.406-411.

Biswas, J., Javachandran, M., Thang, P.V., Fook, V.F.S., Choo, T.S., Qiang, Q., Takahashi, S., Jianzhong, E.H., Feng, C.J. and Kiat, P.Y.L. (2006a) 'Agitation monitoring of persons with dementia based on acoustic sensors, pressure sensors and ultrasound sensors: A feasibility study', In 'Promoting Independence for Older Persons with Disabilities', W.C. Mann and A. Helal (eds.), IOS Press, Fairfax, US.

Biswas, J., Foo, V., Fook, S., Qiang, Q., Jayachandran, M., Wai, A.A.P., Thang, P.V., Htwe, T.M., Siong, L.B. and Yap, P. (2006b) 'Data collection and feature extraction for a smart ward application', Proceedings of the IEEE Conference on Emerging Technologies and Factory Automation, 20-22 September, Prague, 110-115.

Blackman, T., Van Schaik, P. and Martyr, A. (2007) 'Outdoor environments for people with dementia: An exploratory 
study using virtual reality', Ageing society, Vol. 27(6), pp. 811-825.

Bradley, M.M. and Lang, P.J. (1994) 'Measuring emotion: The self-assessment manikin and the semantic differential', Journal of Behavioral Therapy and Experimental Psychiatry, Vol. 25, pp.49-59.

Brouwer, B.G., Visseren, F.L.J. and van der Graaf, Y. (2007) 'The effect of leisure-time physical activity on the presence of metabolic syndrome in patients with manifest arterial disease. The SMART study', American Heart Journal, Vol. 154(6), pp.1146-1152.

Chan, H.K. (2010) 'Agent-based factory level wireless local positioning system with ZigBee technology', IEEE Systems Journal, Vol. 4(2), pp.179-185.

Chen, C.-M. (2011) 'Web-based remote human pulse monitoring system with intelligent data analysis for home health care', Expert Systems with Applications, Vol. 38, pp.2011-2019.

Commissaris, C.J.A.M., Jolles, J., Verhey, F.R.J., Ponds, R.W.H.M., Damoiseaux, V.G.M. and Kok, G.J. (1996) 'Forgetfulness and dementia? Who is worried and why?', European Journal of Public Health, Vol. 6(4), pp.297-299.

Daniel, K.M., Cason, C.L. and Ferrell, S. (2009) 'Emerging technologies to enhance the safety of older people in their homes', Geriatric Nursing, Vol. 30(6), pp.384-389.

De, D., Tang, S., Song, W.-Z., Cook , D. and Das, S.K. (2012) 'ActiSen: Activity-aware sensor network in smart environments', Pervasive and Mobile Computing, doi.10.1016/j.pmcj.2011.12.005.

Domingo, M.C. (2012) 'Throughput efficiency in body sensor networks: A clean-slate approach', Expert Systems with Applications, doi:10.1016/j.eswa.2012.02.182.

Duchêne, F., Garbay, C. and Rialle, V. (2007) 'Learning recurrent behaviours from heterogeneous multivariate time-series', Artificial Intelligence in Medicine, Vol. 39, pp.25-47.

Duffy, J.F., Cain, S.W., Chang, A.-M., Phillips, A.J.K., Münch, M.Y., Gronfier, C., Wyatt, J.K., Dijk, D.-J., Wright, K.P. and Czeisler, C.A. (2011) 'Sex difference in the near-24hour intrinsic period of the human circadian timing system', Proceedings of the National Academy of Sciences (PNAS), Vol. 108(3), pp.15602-15608.

Eisenstein, E.M., Bonheim, P. and Eisenstein, D. (1994) 'Habituation of the galvanic skin response to tone as a function of age', Brain Research Bulletin, Vol. 37(4), pp.343-350.

Ellaway, P.H., Kuppuswamy, A., Nicotra, A. and Mathias, C.J. (2010) 'Sweat production and the sympathetic skin response: Improving the clinical assessment of autonomic function', Autonomic Neuroscience: Basic and Clinical, Vol. 155, pp.109-114.

Engelhardt, W.J. (1999) 'The direct economic costs of insomnia in the United States for 1995', Sleep, Vol. 22(2), pp.S386S393.

Farella, E., Falavigna, M. and Riccò, B. (2010) 'Aware and smart environments: The Casattenta project', Microelectronics Journal, Vol. 41, pp.697-702.

Fei, D.-Y., Zhao, X., Boanca, C., Hughes, E., Bai, O., Merrell, R. and Rafiq, A. (2010) 'A biomedical sensor system for realtime monitoring of astronauts' physiological parameters during Extra-Vehicular Activities', Computers in Biology and Medicine, Vol. 40, pp.635-642.

Fenza, G., Furno, D., and Loia, V. (2012) 'Hybrid approach for context-aware service discovery in healthcare domain',
Journal of Computer and System Sciences, doi:10.1016/j.jcss.2011.10.011.

Gao, J., Xiao, Y., Liu, J., Liang, W. and Chen, C.L.P. (2012) 'A survey of communication/networking in smart grids', Future Generation Computer Systems, Vol. 28, pp.391-404.

Gulbandilar, E., Cimbiz, A., Sari, M. and Ozden, H. (2008) 'Relationship between skin resistance level and static balance in type II diabetic subjects', Diabetes Research and Clinical Practice, Vol. 82, pp.335-339.

Heerink, M., Kröse, B., Evers, V. and Wielinga, B. (2008) 'The influence of social presence on acceptance of a companion robot by older people.' Journal of Physical Agents, Vol. 2(2), pp.33-40.

Heerink, M., Krose, B., Evers, V. and Wielinga, B. (2009) 'Measuring acceptance of an assistive social robot: a suggested toolkit', Proceedings of the 18th IEEE International Symposium on Robot and Human Interactive Communication (RO-MAN 2009), 27th September-2nd October, Toyama, Japan.

Ho, W.C., Hartley, W.R., Myers, L., Lin, M.H., Lin, Y.-S., Lien, C.-H. and Lin, R.-S. (2007) 'Air pollution, weather, and associated risk factors related to asthma prevalence and attack rate', Environmental Research, Vol. 104, pp.402-409.

Hsu, M.-H., Yeh, Y.-T., Chen, C.-Y., Liu, C.-H. and Liu, C.-T. (2011) 'Online detection of potential duplicate medications and changes of physician behavior for outpatients visiting multiple hospitals using health insurance smart cards in Taiwan', International Journal of Medical Informatics, Vol. 80, pp.181-189.

Hu, B., Majoe, D., Ratcliffe, M., Qi, Y., Zhao, Q., Peng, H., Fan, D., Zheng, F., Jackson, M. and Moore, P. (2011) 'EEGbased cognitive interfaces for ubiquitous applications: Developments and challenges', IEEE Intelligent Systems, September/October, pp.46-53.

Jiang, Y., Hamada, H., Shiono, S., Kanda, K., Fujita, T., Higuchi, K. and Maenaka, K. (2010) 'A PVDF-based flexible cardiorespiratory sensor with independently optimized sensitivity to heartbeat and respiration', Procedia Engineering, Vol. 5, pp.1466-1469.

Johansson, C. and Skoog, I. (1996) 'A population-based study on the association between dementia and hip fractures in 85year olds', Aging (Milan), Vol. 8(3), pp.189-196.

Kaye, J. (2008) 'Home-based technologies: A new paradigm for conducting dementia prevention trials', Alzheimer's \& Dementia, Vol. 4, pp.S60-S66.

Khawandi, S., Daya, B. and Chauvet, P. (2011) 'Implementation of a monitoring system for fall detection in elderly healthcare', Procedia Computer Science, Vol.3, pp.216-220.

Klasnja, P. and Pratt, W. (2012) 'Healthcare in the pocket: Mapping the space of mobile-phone health interventions', Journal of Biomedical Informatics, Vol. 45, pp.184-198.

Klerman, E.B. and Dijk, D.J. (2008) 'Age-related reduction in the maximal capacity for sleep - implications for insomnia', Current Biology, Vol. 18, pp.1118-1123.

Kloss, J.D., Szuba, M.P. and Dinges, D.F., in Davis, K.L., Charney, D., Coyle, J.T. and Nemeroff, C. (eds.) (2002) 'Neuropsychopharmacology: The fifth generation of progress', Chapter 130, American College of Neuropsychopharmacology, Lippincott, Williams and Wilkins, Philadelphia, pp.1895-1905.

Kool, B., Ameratunga, S., Robinson, E. and Jackson, R. (2007) 'Hospitalisations and deaths due to unintentional falls at 
home among working-aged New Zealanders', International Journal of the Care of the Injured, Vol. 38, pp.570-575.

Kool, B., Ameratunga, S., Robinson, E., Crengle, S. and Jackson, R. (2008) 'The contribution of alcohol to falls at home among working-aged adults', Alcohol, Vol. 42, pp.383-388.

Kripke, D.F., Garfinkel, L., Wingard, D.L., Klauber, M.R. and Marler, M.R. (2002) 'Mortality associated with sleep duration and insomnia', Archive of General Psychiatry, Vol. 59, pp.131-136.

Krishnamurti, T., Schwartz, D., Davis, A., Fischhoff, B., de Bruin, W.B., Lave, L. and Wang, J. (2012) 'Preparing for smart grid technologies: A behavioral decision research approach to understanding consumer expectations about smart meters', Energy Policy, Vol. 41, pp.790-797.

Lee, Y.D. and Chung, W.Y. (2009) 'Wireless sensor network based wearable smart shirt for ubiquitous health and activity monitoring', Sensors and Actuators B: Chemical, Vol. 140(2), pp.390-395.

Leon, E., Clarke, G., Callaghan, V. and Sepulveda, F. (2007) 'A user-independent real-time emotion recognition system for software agents in domestic environments', Engineering Applications of Artificial Intelligence, Vol. 20, pp.337-345.

Li, Y., Tong, S., Liu, D., Gai, Y., Wang, X., Wang, J., Qiu, Y. and Zhu, Y. (2008) 'Abnormal EEG complexity in patients with schizophrenia and depression', Clinical Neurophysiology, Vol. 119, pp.1232-1241.

Lin, C.-C., Chiu, M.-J., Hsiao, C.-C., Lee, R.-G. and Tsai, Y.-S. (2006) 'Wireless health care service system for elderly with dementia', IEEE Transactions on Information Technology in Biomedicine, Vol. 10(4), pp.696-704.

López-Nores, M., Blanco-Fernández, Y., Pazos-Arias, J.J. and García-Duque, J. (2012) 'The iCabiNET system: Harnessing Electronic Health Record standards from domestic and mobile devices to support better medication adherence', Computer Standards \& Interfaces, Vol. 34, pp.109-116.

Marschollek, M. and Demirbilek, E. (2006) 'Providing longitudinal health care information with the new German Health Card A pilot system to track patient pathways', Computer Methods and Programs in Biomedicine, Vol. 81, pp.266271.

Mathie, M.J., Basilakis, J. and Celler, B.G. (2001) 'A system for monitoring posture and physical activity using accelerometers', 23rd Annual International Conference of the IEEE Engineering in Medicine and Biology Society, October 25-28, Istanbul, Turkey.

Miskelly, F. (2004) 'A novel system of electronic tagging in patients with dementia and wandering', Age and Ageing, Vol. 33, pp.304-306.

Nagai, Y., Goldstein, L.H., Critchley, H.D. and Fenwick, P.B.C. (2004) 'Influence of sympathetic automatic arousal on cortical arousal: Implications for a therapeutic behavioural intervention in epilepsy', Epilepsy Research, Vol. 58, pp.185-193.

Naszlady, A. and Naszlady, J. (1998) 'Patient health record on a smart card', International Journal of Medical Informatics, Vol. 48, pp.191-194.

Pandian, P.S., Mohanavelu, K., Safeer, K.P., Kotresh, T.M., Shakunthala, D.T., Gopal, P. and Padaki, V.C. (2008) 'Smart Vest: Wearable multi-parameter remote physiological monitoring system', Medical Engineering and Physics, Vol. 30, pp.466-477.

Papousek, I. and Schulter, G. (2001) 'Associations between EEG asymmetries and electrodermal lability in low vs. high depressive and anxious normal individuals', International Journal of Phychophysiology, Vol. 41, pp.105-117.

Pasman, E.P., Murnaghan, C.D., Bloem, B.R. and Carpenter, M.G. (2011) 'Balance problems with Parkinson's Disease: Are they anxiety-dependent?', Neuroscience, Vol. 177, pp.283291.

Perlovsky, L.I. (2006) 'Toward physics of the mind: Concepts, emotions, consciousness, and symbols', Physics of Life Reviews, Vol. 3, pp.23-55.

Peter, C. and Herbon, A. (2006) 'Emotion representation and physiology assignments in digital systems', Interacting with Computers, Vol. 18, pp.139-170.

Pichler, M., Schwarzer, S., Stelzer, A. and Vossiek, M. (2009) 'Multi-channel distance measurement with IEEE 802.15.4 (ZigBee) devices', IEEE Journal of Selected Topics in Signal Processing, Vol. 3(5), pp.845-859.

Pullimood, T.B., Corden, J.M., Bryden, C., Sharples, L. and Nasser, S.M. (2007) 'Epidemic asthma and the role of the fungal mold alternaria alternata', Journal of Allergy and Clinical Immunology, Vol. 120(3), pp.610-617.

Roberts, C. and Mort, M. (2009) 'Reshaping what counts as care: Older people, work and new technologies', European Journal of Disability Research, Vol. 3, pp.138-158.

Rodrigues, J.J.P.C., Pereira, O.R.E. and Neves, P.A.C.S. (2011) 'Biofeedback data visualization for body sensor networks', Journal of Network and Computer Applications, Vol. 34, pp.151-158.

Røeggen, I., Storm, H. and Harrison, D. (2011) 'Skin conductance variability between and within hospitalised infants at rest', Early Human Development, Vol. 87, pp.37-42.

Rowe, M.A., Kelly, A., Horne, C., Lane, S., Campbell, J., Lehman, B., Phipps, C., Keller, M. and Benito, A.P. (2009) 'Reducing dangerous nighttime events in persons with dementia by using a nighttime monitoring system', Alzheimer's and Dementia, Vol. 5, pp.419-426.

Santhi, N., Thorne, H.C., van der Veen, D.R., Johnsen, S., Mills, S.L., Hommes, V., Schlangen, L.J.M., Archer, S.N. and Dijk, D.J. (2011) 'The spectral composition of evening light and individual differences in the suppression of melatonin and delay of sleep in humans', Journal of Pineal Research, Doi:10.1111/j.1600-079X.2011.00970.x, pp.1-13.

Sardini, E. and Serpelloni, M. (2010) 'Instrumented wearable belt for wireless health monitoring', Procedia Engineering, Vol. 5, pp.580-583.

Scanlan, J.M., Brush, M., Quijano, C. and Borson, S. (2002) 'Comparing clock tests for dementia screening: naïve judgements vs formal systems - what is optimal?', International Journal of Geriatric Psychiatry, Vol. 17, pp.1421.

Schelter, B., Winterhalder, M., Maiwald, T., Brandt, A., Schad, A., Schulze-Bonhage, A. and Timmer, J. (2006) 'Testing statistical significance of multivariate time series analysis techniques for epileptic seizure prediction', Chaos, Vol. 16(1), pp.1-10.

Shaw, F. E. (2003) 'Falls in older people with dementia', Geriatrics and Ageing, Vol. 6(7), pp.37-40.

Shaw, F.E. and Kenny, R.A. (1998) 'Can falls in patients with dementia be prevented?', Age and Ageing, Vol. 27, pp.7-9.

Soller, B.R., Cabrera, M., Smith, S.M. and Sutton, J.P. (2002) 'Smart medical systems with application to nutrition and fitness in space', Nutrition, Vol. 18, pp.930-936.

Steele, R., Lo, A., Secombe, C. and Wong, Y.K. (2009) 'Elderly persons' perception and acceptance of using wireless sensor 
networks to assist healthcare', International Journal of Medical Informatics, Vol. 78, pp.788-801.

Steiger, A. and Kimuru, M. (2010) 'Wake and sleep EEG provide biomarkers in depression', Journal of Psychiatric Research, Vol. 44, pp.242-252.

Stroulia, E., Chodos, D., Boers, N.M., Huang, J., Gburzynski, P. and Nikolaidas, I. (2009) 'Software engineering for health education and care delivery systems: The Smart Condo project', Proceedings of SEHC'09, 18-19 May, Vancouver, Canada, pp.20-28.

Thomas, A.M., Moore, P., Shah, H., Evans, C., Sharma, M., Xhafa, F., Mount, S., Pham, H.V., Wilcox, A.J., Patel, A., Chapman, C. and Chima, P. (2013) 'Smart care spaces: Needs for intelligent at-home care', International Journal of Space-Based and Situated Computing, Vol. 3(1), pp.35-44.

van Bemmel, A.L. (1996) 'The link between sleep and depression: The effects of antidepressants on EEG sleep', Journal of Psychosomatic Research, Vol. 42(6), pp.555-564.

van der Broek, E.L. and Westerink, J.H.D.M. (2009) 'Considerations for emotion-aware consumer products', Applied Ergonomics, Vol. 40, pp.1055-1064.

Vidal, M., Turner, J., Bulling, A. and Gellersen, H. (2011) 'Wearable eye tracking for mental health monitoring', Computer Communications, doi:10.1016/j.comcom.2011. 11.002.

Vinciarelli, A., Pantic, M. and Bourland, H. (2009) 'Social signal processing: Survey of an emerging domain', Image and Vision Computing, Vol. 27, pp.1743-1759.

Virone, G. (2009) 'Assessing everyday life behavioral rhythms for the older generation', Pervasive and Mobile Computing, Vol. 5, pp.606-622.

Wang, T. (2010) 'Development direction of smart home systems', Proceedings of the 2nd International Conference on Industrial Mechatronics and Automation, 30-31 May, Wuhan, China, pp.310-313.

Wood, G. and Newborough, M. (2003) 'Dynamic energyconsumption indicators for domestic appliances: environment, behaviour and design', Energy and Buildings, Vol. 35, pp.821-841.

Wu, P. and Miller, C. (2005) 'Results from a field study: The need for an emotional relationship between the elderly and their assistive technologies.' 1st International Conference on Augmented Cognition, Las Vegas, USA.

Yanfen, L. and Pu, H. (2011) 'Smart sportswear', Proceedings of the International Conference on Future Computer Science and Education, Xi'an, China, 20-21 August 2011, pp.135138.

Yang, Y., Wei, Z., Jia, D., Cong, Y. and Shan, R. (2010) 'A cloud architecture based on smart home', Proceedings of the Second International Workshop on Education Technology and Computer Science, 6-7 March, Wuhan, China, pp.440443.

Zeisel, J., Silverstein, N.M., Hyde, J., Levkoff, S., Lawton, M.P. and Holmes, W. (2003) 'Environmental correlates to hehavioral health outcomes in Alzheimer's special care units', The Gereontologist, Vol. 43(5), pp.697-711.

Zhang, S., Hu, S., Chao, H.H., Luo, X., Farr, O.M. and Li, C.-S.R. (2012) 'Cerebral correlates of skin conductance responses in a cognitive task', NeuroImage, Vol. 62, pp.1489-1498. 